\title{
Strangeness and charm content of the nucleon
}

\author{
Gunnar Bali*, Sara Collins and Andreas Schäfer \\ Institut für Theoretische Physik, Universität Regensburg, \\ 93040 Regensburg, Germany \\ E-mail: gunnar.baliephysik.uni-regensburg.de, \\ sara.collinsephysik.uni-regensburg.de, \\ andreas.schaeferephysik.uni-regensburg.de
}

(QCDSF Collaboration)

\begin{abstract}
We present results on the scalar strangeness and charm contents of the nucleon and of the disconnected contributions to the nucleon spin. These are obtained on $n_{F}=2$ non-perturbatively improved Sheikholeslami-Wilson configurations at a pseudoscalar mass of $290 \mathrm{MeV}$. We quote $f_{T_{s}}=m_{s}\langle N|\bar{s} s| N\rangle / m_{\mathrm{N}}=0.070(22)$ as our preliminary value for the strange quark fraction of the nucleon mass and an $\overline{M S}$ scheme value $\Delta s=-0.015(10)$, with as yet unknown systematics, for the strangeness contribution to the spin.
\end{abstract}

The XXVI International Symposium on Lattice Field Theory - LAT2009

July 26-31 2009

Peking University, Beijing, China

\footnotetext{
* Speaker.
} 


\section{Introduction}

We calculate disconnected scalar matrix elements $\langle N|\bar{q} q| N\rangle$ as well as contributions to the spin of the nucleon $\Delta q$. Of particular interest are currents containing the strange quark $q=s$, where only quark-line disconnected diagrams contribute but we also consider heavier and lighter flavours.

The scalar matrix element determines the coupling of the Standard Model Higgs boson to the quarks within the proton. This then might couple to heavy particles that could be discovered in future LHC experiments, some of which are dark matter candidates [i]. The fraction of the proton mass $m_{\mathrm{N}}$ carried by the quark flavour $q, f_{T_{q}}=m_{q}\langle N|\bar{q} q| N\rangle / m_{\mathrm{N}}$, is particularly important since the combination $m_{\mathrm{N}} \sum_{q} f_{T_{q}}$ will appear quadratically in this cross section. It is evident that $f_{T_{q}} \rightarrow 0$ for $m_{q} \rightarrow 0$ and $f_{T_{q}} \propto\langle N|G G| N\rangle / m_{\mathrm{N}}=f_{T_{G}}$ for $m_{q} \rightarrow \infty$. We calculate the scalar matrix element for quark masses up to the charm quark to confirm this limiting behaviour. It is also not immediately obvious whether the charm quark should be considered as a sea quark or not. We remark that in general there will be mixing between the dimension four operators $G G$ and $m_{q} \bar{q} q$. This deserves future study.

Disconnected contributions to the nucleon structure are also needed with respect to precision measurements of Standard Model parameters in $p p$ collisions at the LHC where for instance the resolution of a (hypothetical) mass difference between the $W^{+}$and $W^{-}$bosons is limited by the theoretical knowledge of the asymmetries between up and down as well as between strange and charm sea quark contents of the proton [2].

The spin of the nucleon can be factorized into a quark spin contribution $\Delta \Sigma$, a quark angular momentum contribution $L_{q}$ and a gluonic contribution (spin and angular momentum) $\Delta G$ :

$$
\frac{1}{2}=\frac{1}{2} \Delta \Sigma+L_{q}+\Delta G
$$

In the naïve non-relativistic $\mathrm{SU}(6)$ quark model, $\Delta \Sigma=1$, with vanishing angular momentum and gluon contributions. In this case there will also be no strangeness contribution $\Delta s$ in the factorization,

$$
\Delta \Sigma=\Delta d+\Delta u+\Delta s+\cdots,
$$

where in our notation $\Delta q$ contains both, the spin of the quarks $q$ and of the antiquarks $\bar{q}$. Experimentally, $\Delta s$ is obtained by integrating the strangeness contribution to the spin structure function $g_{1}$ over the momentum fraction $x$. The integral over the range in which data exists $(x \gtrsim 0.004)$ usually agrees with zero. For instance a recent Hermes measurement in the region $x \geq 0.02$ yields [3] $\Delta s=0.037(19)(27)$. This means that non-zero results rely on extrapolations into the experimentally un-probed region of very small $x$ and are model dependent [4, 5]. The standard Hermes analysis [6] yields $\Delta s=-0.085(13)(8)(9)$ in the $\overline{M S}$ scheme.

We reported first results and developed the necessary methods in refs. [7]-9]. These algorithmic studies were performed on rooted staggered $n_{F} \stackrel{?}{=} 2+1$ configurations and indicated a value, $\Delta s>-0.02$. However, there are unresolved theoretical issues with this fermion approach [10], so that for the physics study, of which we present preliminary results here, we employ improved Wilson fermions that have a meaningful continuum limit. 


\section{Simulation details}

Simulations were performed on $24^{3} \times 48$ and $32^{3} \times 64$ QCDSF configurations of $n_{F}=2$ nonperturbatively improved clover Wilson fermions with Wilson gauge action at $\beta=5.29$ and $\kappa_{\text {sea }}=$ 0.13632 . These values correspond to a pseudoscalar mass $m_{\mathrm{PS}} \approx 290 \mathrm{MeV}$ and a lattice spacing $a^{-1} \approx 2.59 \mathrm{GeV}$ [11]. The scale was set from the value $r_{0}^{-1} \approx 422 \mathrm{MeV}$, obtained by chirally extrapolating the combination $m_{\mathrm{N}} r_{0}$ to the physical point. The spatial lattice extents correspond to $L a \approx 1.83 \mathrm{fm}$ and $L a \approx 2.43 \mathrm{fm}$, with a larger $40^{3} \times 64$ volume $\left(L a \approx 3.04 \mathrm{fm} \approx 4.5 \mathrm{~m}_{\mathrm{PS}}\right)$ still being analyzed. We use a modified version of the Chroma code [12].

We vary the quark mass parameter $\kappa_{\text {loop }}$ of the current insertion as well as that of the nucleon valence quarks $\kappa_{\mathrm{val}}$. In particular we combine the values $\kappa=\kappa_{\text {sea }}=0.13632, \kappa=0.13609$ and $\kappa=\kappa_{\text {strange }}=0.13550$, corresponding to pseudoscalar masses $m_{\mathrm{PS}} \approx 290,440$ and $690 \mathrm{MeV}$, respectively. Additional heavier masses are used for the loop.

$\Delta q$ and $\langle N|\bar{q} q| N\rangle$ are extracted from the ratios of three-point functions to two-point functions (at zero momentum):

$$
R^{\mathrm{dis}}\left(t, t_{\mathrm{f}}\right)=-\frac{\operatorname{Re}\left\langle\Gamma_{2 \mathrm{pt}}^{\alpha \beta} C_{2 \mathrm{pt}}^{\beta \alpha}\left(t_{\mathrm{f}}\right) \sum_{\mathbf{x}} \operatorname{Tr}\left(M^{-1}(\mathbf{x}, t ; \mathbf{x}, t) \Gamma_{\text {loop }}\right)\right\rangle_{c}}{\left\langle\Gamma_{\text {unpol }}^{\alpha \beta} C_{2 \mathrm{pt}}^{\beta \alpha}\left(t_{\mathrm{f}}\right)\right\rangle_{c}} .
$$

For the scalar matrix element we use, $\Gamma_{2 \mathrm{pt}}=\Gamma_{\text {unpol }}:=\left(1+\gamma_{4}\right) / 2$ and $\Gamma_{\text {loop }}=\mathbb{1}$. For $\Delta q$ we calculate the difference between two polarizations: $\Gamma_{2 \mathrm{pt}}=\gamma_{j} \gamma_{5} \Gamma_{\text {unpol }}$ and $\Gamma_{\text {loop }}=\gamma_{j} \gamma_{5}$, where we average over all three possible $j$-orientations. The spin projection operators along the $j$-axis read, $P_{\uparrow \downarrow}=$ $\frac{1}{2}\left(\mathbb{1} \pm i \gamma_{j} \gamma_{5}\right)$, so that in this case, $\Gamma_{2 \mathrm{pt}}=-i\left(P_{\uparrow}-P_{\downarrow}\right) \Gamma_{\text {unpol }}$, where we have traded a factor $i$ against taking the real part, rather than the imaginary part, of the nominator in eq. (2.1). The variance of the above expression is reduced by explicitly using the fact that $\operatorname{Im} \operatorname{Tr}\left(M^{-1} \mathbb{1}\right)=\operatorname{Im} \operatorname{Tr}\left(M^{-1} \gamma_{j} \gamma_{5}\right)=0$.

$C^{\alpha \beta}\left(t_{\mathrm{f}}\right)$ denotes the two-point function of the zero momentum projected proton with sink and source spinor indices $\alpha$ and $\beta$ and positions $t_{\mathrm{f}}$ and $t_{\mathrm{i}}=0$. In the limit of large times, $t_{\mathrm{f}} \gg t \gg 0$, in the axial case,

$$
R^{\mathrm{dis}}\left(t, t_{\mathrm{f}}\right)+R^{\mathrm{con}}\left(t, t_{\mathrm{f}}\right) \longrightarrow \Delta q,
$$

where we have not computed the connected contribution $R^{\text {con }}$. This vanishes for strangeness and charm contents. In the scalar case the vacuum contribution $\langle 0|\bar{q} q| 0\rangle=-\sum_{\mathbf{x}} \operatorname{Re}\left\langle\operatorname{Tr}\left(M^{-1}(\mathbf{x}, t ; \mathbf{x}, t)\right\rangle_{c}\right.$ needs to be subtracted from eq. (2.1). We employ sink and source smeared two point functions such that this asymptotic limit is effectively reached for $t=4 a \approx 0.3 \mathrm{fm}$ and $t_{\mathrm{f}}=8 a \approx 0.61 \mathrm{fm}$. We vary $t_{\mathrm{f}}$ to check this assumption and compute the final results from the $t_{\mathrm{f}} \geq 8 a$ data.

The clover Wilson operator $M$ can be written as $2 \kappa M=\mathbb{1}-\kappa D$. We estimate $\operatorname{Tr}\left[M^{-1} \Gamma\right]$ stochastically at the cost of less than 100 preconditioned CG solves on each configuration. The noise is reduced by calculating the first two terms of the hopping parameter expansion explicitly, which corresponds to multiplying the estimates by $(\kappa D)^{2}$. For details, see [9] and references therein.

For the calculation of $\Delta q$ we employ the truncated solver method [7, 9] where for the strange mass on the $32^{3} \times 64$ lattice, $N_{1}=730, N_{2}=50, n_{\mathrm{t}}=40$. For the truncated solves we use the smoothly converging even-odd preconditioned CG algorithm while for the runs to full convergence we employ the faster BiCGstab2 algorithm. For the scalar matrix element we only use these 50 


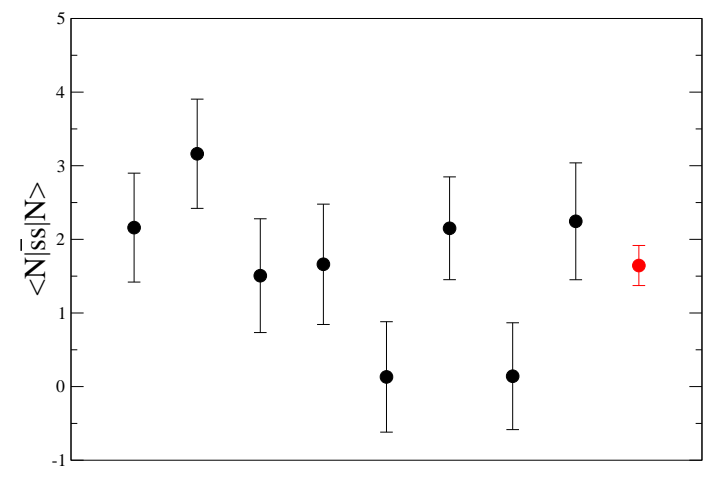

Figure 1: The scalar matrix element at $\kappa_{\text {loop }}=$ $\kappa_{\mathrm{val}}=\kappa_{\text {strange }}$ for 8 different sources and the averaged result.

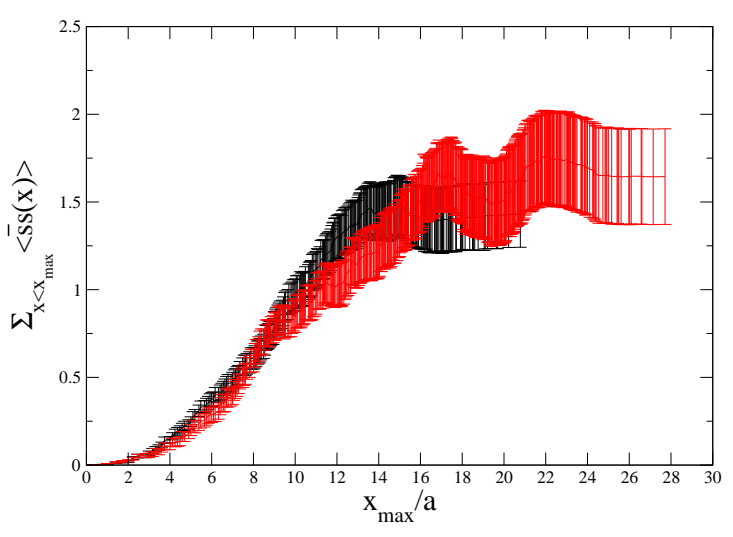

Figure 2: The partial sum eq. (2.3) for the scalar matrix element at $\kappa_{\text {loop }}=\kappa_{\text {val }}=\kappa_{\text {strange }}$ for $L=$ 32 (red symbols) and $L=24$ (black symbols).

solutions since this is dominated by the gauge noise, not by the stochastic noise. We deviate from ref. [9] by seeding the stochastic sources on 8 timeslices rather than on one. This does not cause any computational overhead. We then compute the standard point-to-all propagators that are needed for the nucleon two point functions for 4 time-separated source points on each configuration (requiring 48 solves rather than 12). Exploiting forward and backward propagation in time (replacing $\Gamma_{2 \mathrm{pt}}=\left(1+\gamma_{4}\right) / 2$ by $\left.\left(1-\gamma_{4}\right) / 2\right)$, this then gives us 8 two point (and three point) functions per configuration. Indeed, this averaging reduces the errors of $\Delta s$ and $\langle N|\bar{s} s| N\rangle$ by factors $\sim 1 / \sqrt{8}$ which is the maximal possible gain. We display this for the latter example in figure 1 .

In the ongoing analysis of the $40^{3} \times 64$ volumes we will compute the lowest eigenmodes of the Hermitian Dirac operator $\gamma_{5} M$, to further reduce the stochastic noise of the disconnected loops [9], to precondition the solver and for low mode averaging of the nucleon two point functions [13].

In the present study of the $L=24$ and $L=32$ lattices, where no zero momentum projection is performed at the source, we find it worthwhile to rearrange the nominator within eq. (2.1):

$$
\sum_{|\mathbf{x}| \leq x_{\max }}\left\langle\sum_{\mathbf{y}} C_{2 \mathrm{pt}}\left(\mathbf{y}, t_{\mathrm{f}} ; \mathbf{0}, 0\right) \operatorname{Tr}\left[M^{-1}(\mathbf{x}, t ; \mathbf{x}, t) \Gamma\right]\right\rangle_{c}=\sum_{|\mathbf{x}| \leq x_{\max }} f(\mathbf{x}) \sim x_{\max }^{3} e^{-m x_{\max }}+\cdots
$$

At large $x$ the $f(x) \sim e^{-m x}$ values will eventually not contribute to the signal anymore but just increase the statistical noise. We display the partial sums for the $1.8 \mathrm{fm}(L=24)$ and $2.4 \mathrm{fm}(L=32)$ lattices for the scalar matrix element at $\kappa_{\mathrm{val}}=\kappa_{\text {loop }}=\kappa_{\text {strange }}$ in figure 2. Indeed, at small $x_{\max }$ we see the expected $x_{\max }^{3}$ volume scaling. This flattens somewhat around $x_{\max } \approx 8 a$ but only saturates when the boundaries of the box are hit $\left(x_{\max }=12 a\right.$ and $x_{\max }=16 a$, respectively). Beyond these distances only the lattice "corners" are summed up. This non-saturation means that partial sums can only become a permissible method of reducing the noise at much larger spatial volumes. It also indicates that finite size effects might still be substantial for the $2.4 \mathrm{fm}$ data. The partial sums appear to saturate even more slowly for the case of $\Delta s$.

\section{Results}

In figure 3 we display our $L a \approx 1.8 \mathrm{fm}$ results on $f_{T_{q}}$ for two different nucleon valence quark 


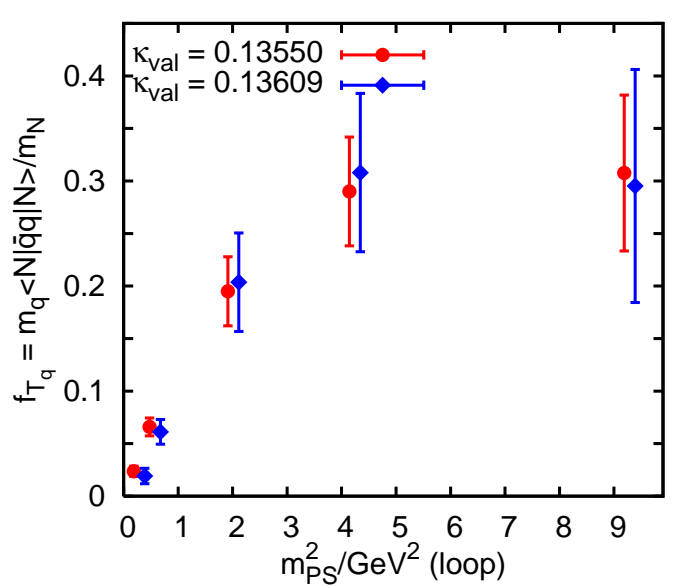

Figure 3: The proton mass fractions $f_{T_{q}}$ for quarks $q$ of different masses.

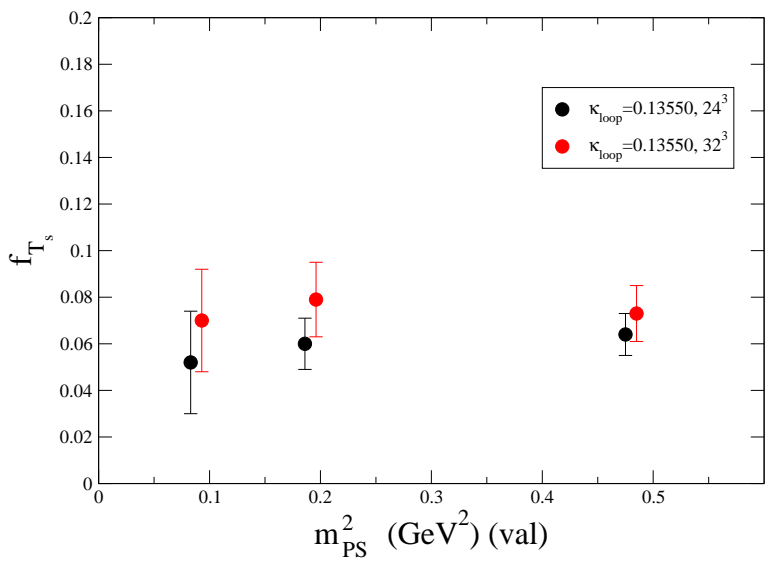

Figure 4: The strangeness $f_{T_{s}}$ for different valence quark masses and volumes.

masses $\kappa=\kappa_{\text {strange }}$ (red symbols) and $\kappa=0.13609$ (blue symbols), as functions of the quark mass $m_{q} \propto m_{\mathrm{PS}}^{2}$ in the loop. The scheme independent combination $m_{q}\langle N|\bar{q} q| N\rangle$ encodes the contribution of the mass $m_{q}$ of quark $q$ to the nucleon mass. This suggests the normalization with respect to the nucleon mass $m_{\mathrm{N}}$. We note that differences between the PCAC and the bare quark masses $m_{q}=\left(\kappa^{-1}-\kappa_{c, \text { val }}^{-1}\right) /(2 a)$, where $\kappa_{c, \text { val }}$ is obtained from an extrapolation of $m_{\mathrm{PS}}^{2}$ to zero, only exceed the percent level for $m_{q}<m_{s}$. The right-most data correspond to the charm, the data near $m_{\mathrm{PS}}^{2} \approx$ $0.48 \mathrm{GeV}^{2}$ to the strange quark. $f_{T_{q}}$ approaches zero as $m_{q} \rightarrow 0$ and saturates at $f_{T_{q}} \approx 1 / 3$ for $m_{q} \gtrsim(2 / 3) m_{c}$. Obviously the charm, bottom or top quarks cannot each be made responsible for one third of the proton's mass. Moreover, in the heavy quark limit, $\langle N|\bar{q} q| N\rangle \sim\langle N|G G| N\rangle / m_{q}$, so that mixing with the gluonic matrix element needs to be considered carefully.

In figure $\emptyset$ we focus on the volume and quark mass dependence of $f_{T_{s}}$. The chiral behaviour, varying the valence pseudoscalar mass from $690 \mathrm{MeV}$ down to the $290 \mathrm{MeV}$ that correspond to the sea, is well fitted by a constant. So, hopefully, the dependence on the sea quark mass will be weak as well. $f_{T_{s}}$ appears to increase on the larger volume, see also figure 2, but to exclude this to be just a statistical fluctuation, we will have to wait for the $L=40$ analysis. The value obtained on the larger volume for the lightest nucleon mass reads,

$$
f_{T_{s}}=\frac{m_{s}\langle N|\bar{s} s| N\rangle}{m_{\mathrm{N}}}=0.070 \pm 0.022
$$

Other recent direct calculations resulted in the values $f_{T_{s}}=0.34(5)$ for anisotropic $n_{F}=2$ Wilson fermions [14] and $f_{T_{s}}=0.015$ (28) for $n_{F}=2$ overlap fermions, fixed to zero topology [13]. An indirect determination with rooted staggered fermions, combining chiral condensate and nucleon two point function data with the Hellmann-Feynman theorem, suggested $f_{T_{s}}=0.063(11)$ [15].

The valence quark mass and volume dependence of $\Delta s$ is displayed in figure 5. Both appear to be mild, with the tendency of a bigger $-\Delta s$ on the larger volume, in particular for the lightest nucleon mass. We quote the value,

$$
\Delta s^{\text {latt }}=-0.020 \pm 0.013
$$




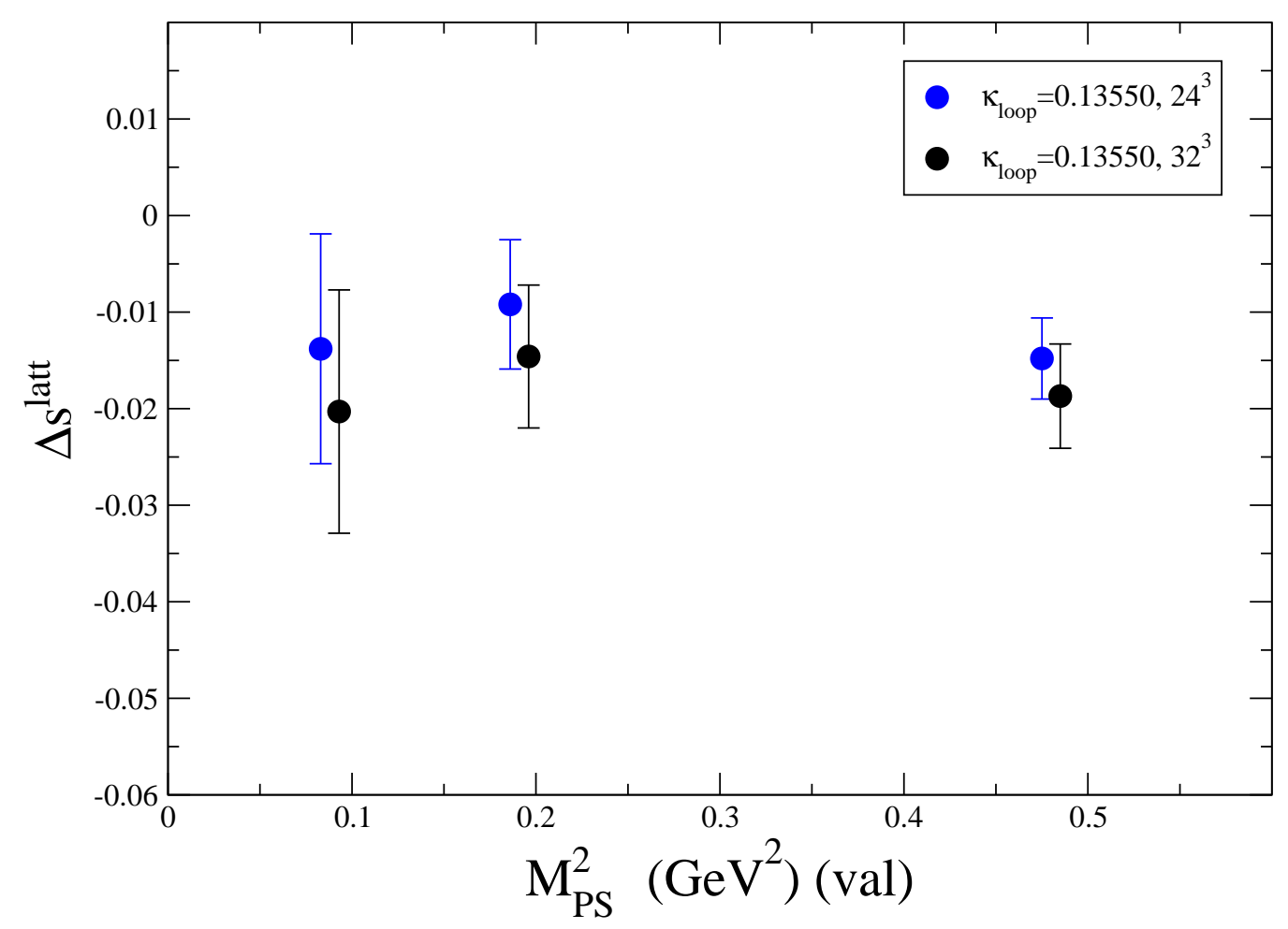

Figure 5: Valence quark mass dependence of $\Delta s^{\text {latt }}$ on the $L a=24 a \approx 1.8 \mathrm{fm}$ and $L a=24 a \approx 2.4 \mathrm{fm}$ lattices.

that we obtain on the large volume for the lightest nucleon mass. Note that this number applies to the lattice scheme and needs to be multiplied by a renormalization factor that we expect to be close to 0.76 for a conversion into the $\overline{M S}$ scheme. Another recent study yielded $\Delta s=-0.0064$ (24) [14], employing $n_{F}=2$ anisotropic Wilson fermions. We remark that our value at $\kappa_{\mathrm{val}}=\kappa_{\text {strange }}$ reads $-0.0187(54)$ and, therefore, differs from zero by 3.5 standard deviations.

\section{Outlook}

At present, the $L a \approx 3 \mathrm{fm} 40^{3} \times 64$ volumes are being analyzed. Disconnected contributions to form factors [16] at non-vanishing momentum transfer and to moments of parton distribution functions are also of big phenomenological interest and will obviously extend the present study. The long term goal of this project is to go to large volumes at the physical sea quark mass.

\section{Acknowledgments}

S. Collins acknowledges support from the Claussen-Simon-Foundation (Stifterverband für die Deutsche Wissenschaft). This work was supported by the EC HadronPhysics2 Integrated Infrastructure Initiative and the DFG Sonderforschungsbereich/Transregio 55. Computations were performed on Regensburg's Athene HPC cluster and on the BlueGene/P (JuGene) and the Nehalem Cluster (JuRoPA) of the Jülich Supercomputer Center. 


\section{References}

[1] J. Ellis, K. A. Olive and P. Sandick, Update on the direct detection of dark matter in MSSM models with non-universal Higgs masses, New J. Phys. 11 (2009) 105015 [arXiv:0905.0107 [hep-ph]].

[2] G. Aad et al. [ATLAS Collaboration], Expected performance of the ATLAS experiment-detector, trigger and physics, arXiv:0901.0512 [hep-ex].

[3] A. Airapetian et al. [HERMES Collaboration], Measurement of parton distributions of strange quarks in the nucleon from charged-kaon production in deep-inelastic scattering on the deuteron, Phys. Lett. B 666 (2008) 446 [arXiv:0803.2993 [hep-ex]].

[4] S. L. Zhu, G. Sacco and M. J. Ramsey-Musolf, Recoil order chiral corrections to baryon octet axial currents and large $N_{c}$ QCD, Phys. Rev. D 66 (2002) 034021 [hep-ex/0201179].

[5] D. de Florian, R. Sassot, M. Stratmann and W. Vogelsang, Extraction of spin-dependent parton densities and their uncertainties, Phys. Rev. D 80 (2009) 034030 [arXiv:0904.3821 [hep-ph]].

[6] A. Airapetian et al. [HERMES Collaboration], Precise determination of the spin structure function $g_{1}$ of the proton, deuteron and neutron, Phys. Rev. D 75 (2007) 012007 [hep-ex/ 0609039 ].

[7] S. Collins, G. Bali and A. Schäfer, Disconnected contributions to hadronic structure: a new method for stochastic noise reduction, POS (LATTICE 2007) 141 [arXiv:0709.3217 [hep-lat].

[8] G. Bali, S. Collins and A. Schäfer, Hunting for the strangeness content of the nucleon, POS (LATTICE 2008)161 arXiv:0811.0807 [hep-lat].

[9] G. S. Bali, S. Collins and A. Schäfer, Effective noise reduction techniques for disconnected loops in Lattice QCD, arXiv:0910.3970 [hep-lat].

[10] M. Creutz, The evil that is rooting, Phys. Lett. B 649 (2007) 230 [hep-lat/ 0701018 .

[11] M. Göckeler et al. [QCDSF and UKQCD Collaborations], Non-perturbative renormalization of three-quark operators, Nucl. Phys. B 812 (2009) 205 [arXiv:0810.3762 [hep-lat]].

[12] R. G. Edwards and B. Joó [LHP and UKQCD Collaborations], The Chroma software system for Lattice QCD, Nucl. Phys. Proc. Suppl. 140 (2005) 832 [hep-lat/ 0409003 ;; C. McClendon, Optimized Lattice QCD kernels for a Pentium 4 cluster, Jlab preprint (2001) JLAB-THY-01-29, http://www.jlab.org/ edwards/qcdapi/reports/dslash_p4.pdf; P.A. Boyle, http://www.ph.ed.ac.uk/ paboyle/bagel/Bagel.html(2005).

[13] K. Takeda, S. Aoki, S. Hashimoto, T. Kaneko, T. Onogi and N. Yamada [JLQCD Collaboration], Calculation of nucleon strange quark content with dynamical overlap quarks, POS (LAT2009) 138 [arXiv:0910.5036 [hep-lat].

[14] R. Babich, R. Brower, M. Clark, G. Fleming, J. Osborn and C. Rebbi, [Disco Collaboration], Strange quark content of the nucleon, POS (LATTICE 2008)160 [arXiv:0901.4569 [hep-lat]]; these Proceedings.

[15] D. Toussaint and W. Freeman [MILC Collaboration], The strange quark condensate in the nucleon in 2+1 flavor QCD, Phys. Rev. Lett. 103 (2009) 122002 [arXiv:0905.2432 [hep-lat]].

[16] T. Doi et al. [ $\chi Q C D$ Collaboration], The calculation of nucleon strangeness form factors from $N_{f}=2+1$ clover fermion lattice QCD, POS (LAT2009) 134 [arXiv:0910.2687 [hep-lat]]. 